ierten Todesfälle nicht näher differenziert. Es ist aber davon auszugehen, dass es sich überwiegend um zerebrale Insulte sowie Komplikationen hypertensiver Krisen gehandelt hat. Auch die Gesamtmortalität war bei Teilnehmern, die Marihuana konsumierten, signifikant um den Faktor 1,29 (95\%-KI: 1,03-1,61) erhöht.

Yankey BA et al. Effect of marijuana use on cardiovascular and cerebrovascular mortality: A study using the National Health and Nutrition Examination Survey linked mortality file. Eur J Prev Cardiol. 2017, doi: 10.1177/2047487317723212

\section{Kommentar}

Bislang standen in der Diskussion um die Legalisierung von Marihuana oder Haschisch vor allem die langfristigen zerebralen Folgen im Mittelpunkt. Demgegenüber ist weniger bekannt, dass durch Marihuana nicht nur ein erhöhter Blutdruck, zum Teil auch mit krisenhaften Blutdrucksteigerungen, entstehen kann, sondern dass auch messbare Effekte auf die mittelfristige Mortalität zu verzeichnen sind.

Für die Praxis ist ferner wichtig, dass mit zunehmender Ausdehnung des Marihuana-
Gebrauchs anamnestisch nicht nur Nikotin und Alkohol im Zusammenhang mit der Hypertonie-Diagnostik von Bedeutung sind.

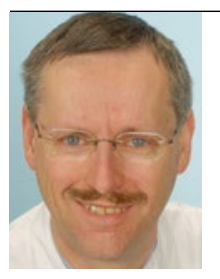

Prof. Dr. med. Walter Zidek Medizinische Klinik IV, Charité Universitätsmedizin Berlin

\title{
Vorhofflimmern und Stentimplantation: Duale Therapie besser als Triple-Therapie
}

\section{Bei Patienten mit Vorhofflimmern, bei denen eine koronare Stent- implantation durchgeführt werden muss, hat die Triple-Therapie mit Marcumar, ASS und Clopidogrel ausgedient.}

$P_{s}^{2}$ atienten mit Vorhofflimmern und Risikofaktoren für einen Schlaganfall benötigen eine effektive orale Antikoagulation. Nach einer elektiven oder auch notfallmäßigen koronaren Stentimplantation ist eine duale Plättchenhemmer-Therapie notwendig. Bei Patienten mit Vorhofflimmern, die sich zudem einer koronaren Stentimplantation unterziehen mussten, wurde daher jahrelang eine Kombination aus einer oralen Antikoagulation und einer dualen Plättchenhemmung empfohlen (TripleTherapie). Hierunter zeigen sich jedoch zum Teil sehr hohe Blutungsraten.
Vor dem Hintergrund der verfügbaren direkten oralen Antikoagulanzien (DOAC) sowie initialen Daten, die den Verzicht einer längeren ASS-Therapie unter oralen Antikoagulanzien in diesem Kontext für möglich erachten (WOESTStudie), wurde die RE-DUAL-Studie konzipiert. In dieser internationalen Studie wurde die bisherige Triple-Therapie (mit Marcumar) gegen eine duale Therapie mit Dabigatran (in beiden Dosierungen, d.h. $2 \times 110 \mathrm{mg}$ und $2 \times 150 \mathrm{mg}$ ) und nur einem Plättchenhemmer (Clopidogrel oder Ticagrelor) nach der Stentimplantation bei Patienten mit Vorhofflim-

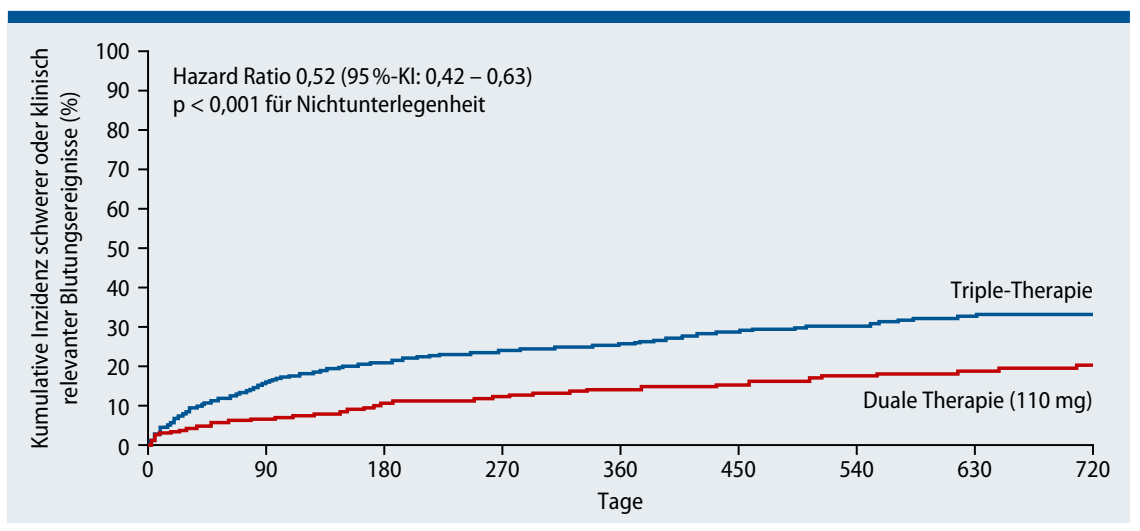

Kaplan-Meier-Analyse des primären Endpunkts (schwere oder klinisch relevante Blutungsereignisse) unter der Dreifachtherapie mit Marcumar oder einer Therapie mit Dabigatran $2 \times 100 \mathrm{mg}$ und Clopidogrel.

mern verglichen. Der primäre Endpunkt war als schwere oder nicht schwere, jedoch klinisch relevante Blutung (z.B. tödliche Blutungen, intrakranielle Blutungen oder Blutungen, die zur Krankenhausaufnahme führen) definiert.

Im Ergebnis zeigte sich ein signifikanter Vorteil der Patienten unter der dualen Therapie mit beiden DabigatranDosen hinsichtlich des primären Endpunkts, wobei es unter der Dosis $2 \times 110$ mg fast zu einer Halbierung schwerer Blutungsereignisse $\mathrm{kam}(15,4 \%$ vs. $26,9 \%$, Hazard Ratio 0,52; $\mathrm{p}<0,001$ für Überlegenheit). Die Inzidenz schwerwiegender thromboembolischer Ereignisse war bei der dualen Therapie nicht höher als bei der Triple-Therapie.

Cannon C et al. Dual Antithrombotic Therapy with Dabigatran after $\mathrm{PCl}$ in Atrial Fibrillation. N Engl J Med. 2017, doi: 10.1056/NEJMoa1708454

\section{Kommentar}

Nach der PIONEER-Studie mit Verwendung von Rivaroxaban zeigen sich nun auch unter einer Volldosis von Dabigatran und Clopidogrel oder Ticagrelor wesentlich geringere Blutungsraten als bei der Triple-Therapie, ohne dass es Anzeichen für deutlich höhere Raten thromboembolischer Ereignisse gab. Die Leitlinien werden diese Daten zukünftig sicher berücksichtigen.

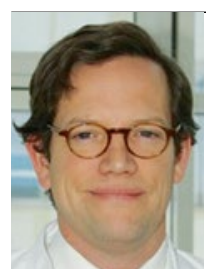

Prof. Dr. med Peter W. Radke Klinik für Innere Medizin Kardiologie, Schön Klinik Neustadt 